\title{
Erratum: Proton-Proton Weak Capture in Chiral Effective Field Theory [Phys. Rev. Lett. 110, 192503 (2013)]
}

\author{
L. E. Marcucci, R. Schiavilla, and M. Viviani \\ (Received 28 May 2019; published 3 July 2019)
}

DOI: 10.1103/PhysRevLett.123.019901

Upon re-examination of the computer programs used for the calculation, we have identified an error in the determination of the $P$-wave reduced matrix elements (RMEs) associated with the longitudinal multipole operator. These RMEs are denoted as $L_{\Lambda}^{L S J}(q)$ in the Letter.

As a consequence of this error, the results relative to the $P$-wave contributions in Tables I and II and in Figs. 2 and 3 are incorrect. In particular, (1) Table I should be replaced by the following table, where we do not list the cumulative, but rather the individual contributions of each partial wave, since the $P$-waves contributions are found to be much smaller than the $S$ wave. (2) Table II should be replaced by the following table, where we have removed the lines corresponding to the $S+P$ waves, since the $P$-waves contributions are negligible. (3) Figures 2 and 3 should be replaced by the following figures, where again we only show the $S$-wave results. (4) The discussions in this Letter of the $P$-wave contributions are changed. In particular, we note the following. (i) On page 3, the sentence "the $P$-wave contributions to $S(0)$ sum up to $\sim 1 \%$ of the total value" should be replaced with "the $P$-wave contributions to $S(0)$ are about 5 orders of magnitude smaller than the $S$-wave ones, and therefore negligible." (ii) The following sentence "the results can be summarized in the conservative range $S(0)=(4.030 \pm 0.006) \times 10^{-23} \mathrm{MeV} \mathrm{fm}^{2}$ " should be replaced with "the results can be summarized in the conservative range $S(0)=(4.008 \pm 0.005) \times 10^{-23} \mathrm{MeV} \mathrm{fm}^{2}$." (iii) The result obtained within the AV18 potential when all the $S$ and $P$ waves are included is again essentially identical to that obtained with the ${ }^{1} S_{0}$ channel only, i.e., $S(0)=(4.000 \pm 0.003) \times 10^{-23} \mathrm{MeV} \mathrm{fm}^{2}$.

In conclusion, the total $P$-wave contributions amount to $\simeq 1.6 \times 10^{-28} \mathrm{MeV} \mathrm{fm}^{2}$, which is in agreement with the recent study of Ref. [2], performed in pionless effective field theory.

There is also an additional issue that has come to light $[3,4]$ since publication of the Letter, i.e., an error in the relation, originally given in Ref. [5], between the low-energy constant (LEC) $c_{D}$ in three-nucleon contact interaction and the LEC $d_{R}$ in the contact two-nucleon axial current. Unfortunately, this error has propagated widely in the nuclear theory community.

TABLE I. Individual $S$ - and $P$-wave contributions to the astrophysical $S$ factor at zero c.m. energy in units of $\mathrm{MeV}^{2} \mathrm{fm}^{2}$ The theoretical uncertainties, given in parentheses, are due to the fitting procedure adopted for the LECs in the weak current and affect essentially only the $S$-wave contributions. The results have been obtained with the two different cutoff values $\Lambda=500$ and $600 \mathrm{MeV}$.

\begin{tabular}{lcccc}
\hline \hline & ${ }^{1} S_{0}$ & ${ }^{3} P_{0}$ & ${ }^{3} P_{1}$ & ${ }^{3} P_{2}$ \\
\hline$\Lambda=500 \mathrm{MeV}$ & $4.008(5) \times 10^{-23}$ & $5.5 \times 10^{-30}$ & $1.2 \times 10^{-28}$ & $3.9 \times 10^{-29}$ \\
$\Lambda=600 \mathrm{MeV}$ & $4.008(5) \times 10^{-23}$ & $5.4 \times 10^{-30}$ & $1.1 \times 10^{-28}$ & $3.9 \times 10^{-29}$ \\
\hline \hline
\end{tabular}

TABLE II. Values for $S^{n}(0) / S(0)$ with $n=1-4$, in units of $\mathrm{MeV}^{-n}$, and the $\chi^{2}$ as defined in the text, obtained with a polynomial fit of $S(E)$ up to orders $O\left(E^{2}\right)$ (Fit 1), $O\left(E^{3}\right)$ (Fit 2), and $O\left(E^{4}\right)$ (Fit 3), retaining only $S$ waves, since the $P$-wave contributions are negligible. Also listed are the results of Ref. [1]. The theoretical uncertainties, listed only for $n=1,2,3$, are given in parentheses and account for the cutoff sensitivity and the errors due to the LEC's fitting procedure.

\begin{tabular}{lcccr}
\hline \hline$n$ & 1 & 2 & 3 & 4 \\
\hline Fit 1 & $12.23(1)$ & $178.4(3)$ & & $\chi^{2}$ \\
Fit 2 & $11.42(1)$ & $239.6(5)$ & $-1464(5)$ & $1.2 \times 10^{-3}$ \\
Fit 3 & $10.82(1)$ & $317.4(6)$ & $-5839(9)$ & $98 \times 10^{3}$ \\
Ref. [1] & $11.3(1)$ & $170(2)$ & & $2.1 \times 10^{-5}$ \\
\hline \hline
\end{tabular}




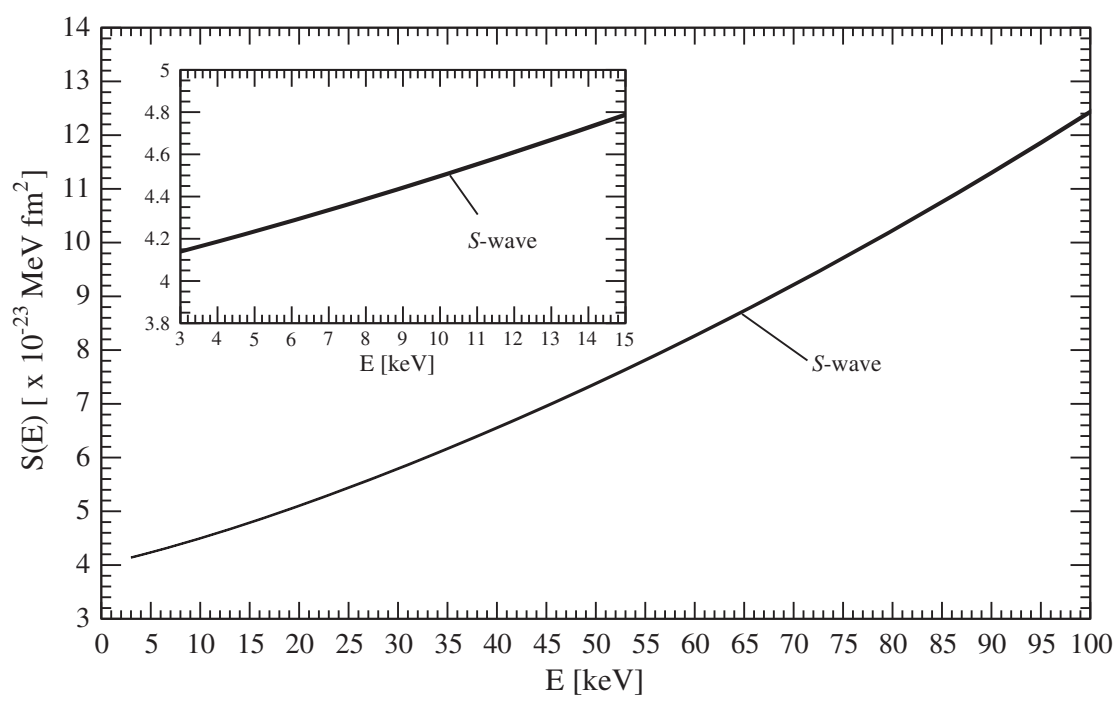

FIG. 1. The astrophysical $S$ factor as function of the c.m. energy in the range $0-100 \mathrm{keV}$. Since the $S$ - and $(S+P)$-wave results essentially overlap, only the $S$-wave results are shown. In the inset, $S(E)$ is shown in the range $3-15 \mathrm{keV}$.

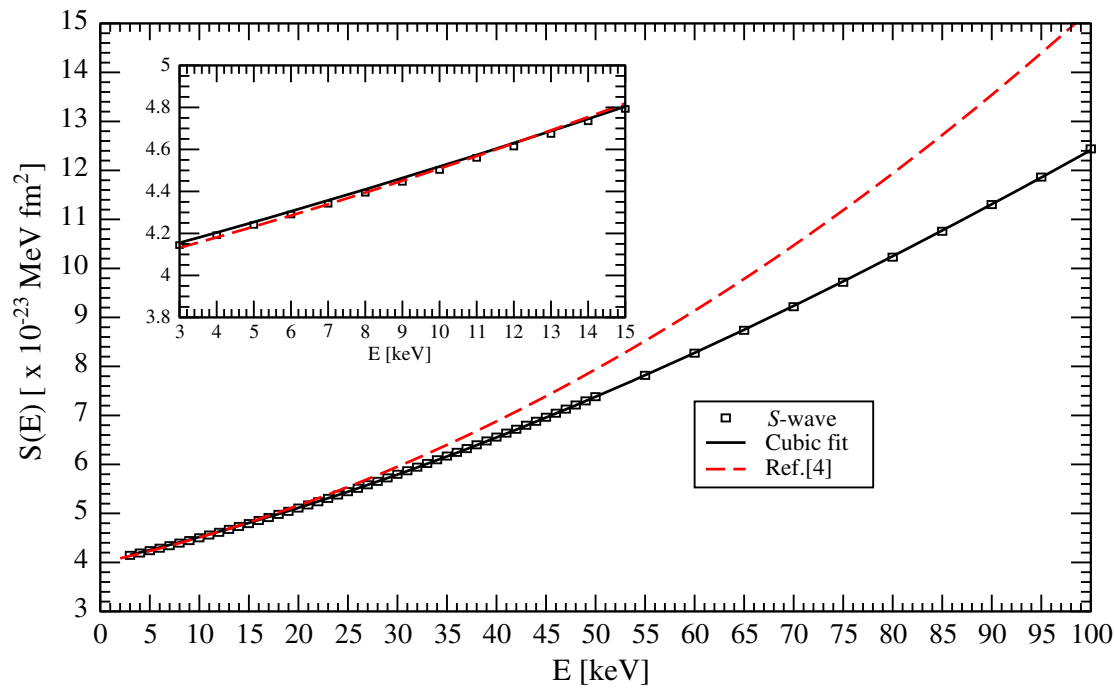

FIG. 2. The astrophysical $S$ factor in the energy range $0-100 \mathrm{keV}$, calculated with $\Lambda=500 \mathrm{MeV}$ and $c_{D}=-0.20$ and including the $S$-wave contributions is compared with the cubic polynomial fit and the quadratic fit of Ref. [1]. In the inset, $S(E)$ is shown in the range 3-15 keV. The $(S+P)$-wave results are not shown, since they would be indistinguishable from the $S$-wave ones.

However, as we noted in Ref. [6], we expect this error to have a negligible impact on the present predictions. In order to test this expectation, as well as to take into account the most recent developments in both nuclear chiral interactions [7-9] and currents [10], we are in the process of re-examining the $p p$ weak capture in toto.

[1] J.-W. Chen, C.-P. Liu, and S.-H. Yu, Phys. Lett. B 720, 385 (2013).

[2] B. Acharya, L. Platter, and G. Rupak, arXiv:1903.02590.

[3] R. Schiavilla (unpublished).

[4] A. Baroni, R. Schiavilla, L. E. Marcucci, L. Girlanda, A. Kievsky, A. Lovato, S. Pastore, M. Piarulli, S. C. Pieper, M. Viviani, and R. B. Wiringa, Phys. Rev. C 98, 044003 (2018).

[5] D. Gazit, S. Quaglioni, and P. Navratil, Phys. Rev. Lett. 103, 102502 (2009).

[6] L. E. Marcucci, A. Kievsky, S. Rosati, R. Schiavilla, and M. Viviani, Phys. Rev. Lett. 121, 049901(E) (2018).

[7] M. Piarulli, L. Girlanda, R. Schiavilla, R. N. Perez, J. E. Amaro, and E. R. Arriola, Phys. Rev. C 91, 024003 (2015). 
[8] M. Piarulli, L. Girlanda, R. Schiavilla, A. Kievsky, A. Lovato, L. E. Marcucci, S. C. Pieper, M. Viviani, and R. B. Wiringa, Phys. Rev. C 94, 054007 (2016).

[9] D. R. Entem, R. Machleidt, and Y. Nosyk, Phys. Rev. C 96, 024004 (2017).

[10] R. Schiavilla, A. Baroni, S. Pastore, M. Piarulli, L. Girlanda, A. Kievsky, A. Lovato, L. E. Marcucci, S. C. Pieper, M. Viviani, and R. B. Wiringa, Phys. Rev. C 99, 034005 (2019). 